\title{
Time-resolved X-ray diffraction reveals the hidden mechanism of high piezoelectric activity in uniaxial ferroelectric
}

\author{
Semën Gorfman ${ }^{1}$, Hyeokmin Choe ${ }^{1}$, Vladimir V. Shvartsman ${ }^{2}$, Michael Ziolkowski ${ }^{1}$, \\ Marco Vogt ${ }^{1}$, Jörg Strempfer ${ }^{3}$, Tadeusz Łukasiewicz ${ }^{4}$, Ullrich Pietsch ${ }^{1}$, Jan Dec $^{5}$ \\ ${ }^{1}$ Department of Physics, University of Siegen, Siegen, Germany \\ ${ }^{2}$ Institute for Materials Science, University of Duisburg-Essen, Essen, Germany \\ ${ }^{3}$ Deutsches Elektronen-Synchrotron DESY, Notkestrasse 85, D-22603 Hamburg, Germany \\ ${ }^{4}$ Institute of Electronic Materials Technology, Warsaw, Poland \\ ${ }^{5}$ Institute of Materials Science, University of Silesia, Katowice, Poland
}

\begin{abstract}
High piezoelectric activity of many ferroelectrics has been the focus of numerous recent studies. The structural origin of this activity remains poorly understood due to a lack of appropriate experimental techniques and mixing of different mechanisms related to ferroelectricity and ferroelasticity. Our work reports on the study of a uniaxial $\mathrm{Sr}_{0.5} \mathrm{Ba}_{0.5} \mathrm{Nb}_{2} \mathrm{O}_{6}$ ferroelectric where the formation of regions with different spontaneous strains is ruled out for the symmetry reason and where the interrelation between piezoelectricity and ferroelectricity can be inspected in the isolated fashion. We performed X-ray diffraction experiment on a single crystalline sample under alternating electric field and observed unknown hidden-in-the-bulk mechanism, which suggests that the highest piezoelectric activity is realized in the volumes where nucleation of small ferroelectric domains takes place. This new mechanism creates a novel roadmap for designing materials with enhanced piezoelectric properties.
\end{abstract}

Electromechanical coupling is the ability of some solids to convert mechanical energy into electrical and vice versa. Solids exhibiting linear electromechanical coupling are called piezoelectrics: they may become electrically polarized under a mechanical stress or mechanically deformed under an electric field. Piezoelectricity closely co-exists with ferroelectricity - the ability to switch spontaneous polarization states under an electric field. Although a piezoelectric material does not have to be ferroelectric, the piezoelectric coefficients of many ferroelectrics exceed those of non-ferroelectrics by remarkable two orders of magnitude [1]. Two mechanisms of piezoelectric activity in ferroelectrics are currently known: intrinsic and extrinsic. The intrinsic mechanism stems from the shifts of a 
charge density and the modification of the bond force balance under external stress / electric field [1-4]. The extrinsic mechanism results from the possibility of domain walls motion and the modification of the volumes possessing different polarization / strain [5-7]. The extrinsic effect can be maximized by domain engineering, while intrinsic piezoelectrics must be designed on the level of atomic structure and chemical bonds. The proper understanding of the piezoelectricity is crucial knowledge for the development of new materials, for example environment friendly alternatives to the dominating $\mathrm{PbZr}_{1-\mathrm{x}} \mathrm{Ti}_{\mathrm{x}} \mathrm{O}_{3}(\mathrm{PZT})$. Despite significant efforts, the ratio of intrinsic and extrinsic contributions remains elusive - mainly because of the limited ability to inspect all the processes involved. For example, piezoresponse force microscopy mainly probes surfaces; transmission electron microscopy addresses nano-meter length-scales; optical methods of domain imaging (e.g. [8,9]) are insensitive to lattice parameters. X-ray and neutron diffraction methods access macroscopic, microscopic and mesoscopic length scales; they have been exploited for understanding of the ferroelectrics and relaxor systems (e.g. discovery of monoclinic phases [10-13], lattice properties of relaxors [14-17], structural disorder [18,19]). However their uses for in-situ probing of piezoelectric activity in ferroelectrics are rare and mainly limited to ceramics / powders [20]. Furthermore, many studies focus on technologically important pseudo-cubic ferroelectrics such as PZT or $\mathrm{PbMg}_{1 / 3} \mathrm{Nb}_{2 / 3} \mathrm{O}_{3}$, where domains share both ferroelectric and ferroelastic features, so that intrinsic and extrinsic contributions mix with each other. In this view, investigation of piezoelectricity of uniaxial ferroelectrics such as $\mathrm{Sr}_{0.5} \mathrm{Ba}_{0.5} \mathrm{Nb}_{2} \mathrm{O}_{6}$ (SBN50) where formation of regions of different spontaneous strains is forbidden by symmetry (the temperature-driven paraelectric-to-ferroelectric phase transition does not change the crystallographic system) may help to inspect the interrelation between the ferroelectricity and piezoelectricity in the well-defined fashion. It is strange but true, that such investigations have received little attention so far.

This letter reports on the observation of a new piezoelectric activity mechanism, suggesting that it may appear in a purely uniaxial ferroelectric in the form of correlation between lattice parameter and domains size. We observed this mechanism by time-resolved synchrotron $\mathrm{X}$ ray diffraction on SBN50 single crystals under alternating electric field. We have chosen SBN50 as a model uniaxial ferroelectric whose para- and ferroelectric phases are tetragonal (c/a 0.31 e.g. [21]), formation of ferroelastic domains is forbidden and static patterns of purely ferroelectric domains are well documented [22-24]. We quantified the electric-field induced strain and demonstrated that the volumes of rapid domain growth / merging during 
the polarization reversal exhibit anomalously high deformation under electric field $(\sim 400$ $\mathrm{pC} / \mathrm{N})$, comparable to that of the PZT $[25,26]$.

The SBN50 single crystal was grown by the Czochralski method [27] and cut to a $0.5 \mathrm{~mm}$ [001] oriented plate; gold electrodes were deposited on both faces in order to apply external high-voltage (HV). We generated $20 \mathrm{~Hz}$ periodic triangular signals reaching $\pm 150 \mathrm{~V}$ (subcoercive (SC) field of $300 \mathrm{~V} / \mathrm{mm}$ ) and $\pm 975 \mathrm{~V}$ (over-coercive (OC) field of $1950 \mathrm{~V} / \mathrm{mm}$ ). Electrical current was measured using an $1 \mathrm{k} \Omega$ active probe and integrated in order to estimate the dielectric polarization (Figure 1). The dielectric response remained stable over the entire measurement time ( 1 week $=10^{7} \mathrm{HV}$ cycles). The SC current / polarization hysteresis can be explained by either the presence of an electric conduction or an irreversible domain wall motion (Rayleigh effect) [28-30]. The OC current shows characteristic ferroelectric switching behaviour with the maxima at $786 \mathrm{~V} / \mathrm{mm}$ and $-634 \mathrm{~V} / \mathrm{mm}$ for the rising and falling voltage. The asymmetry of P-E loops clearly indicates that these two polarization reversals may be driven by different mechanisms; such asymmetries are commonly observed in SBN (e.g. [31,32]).

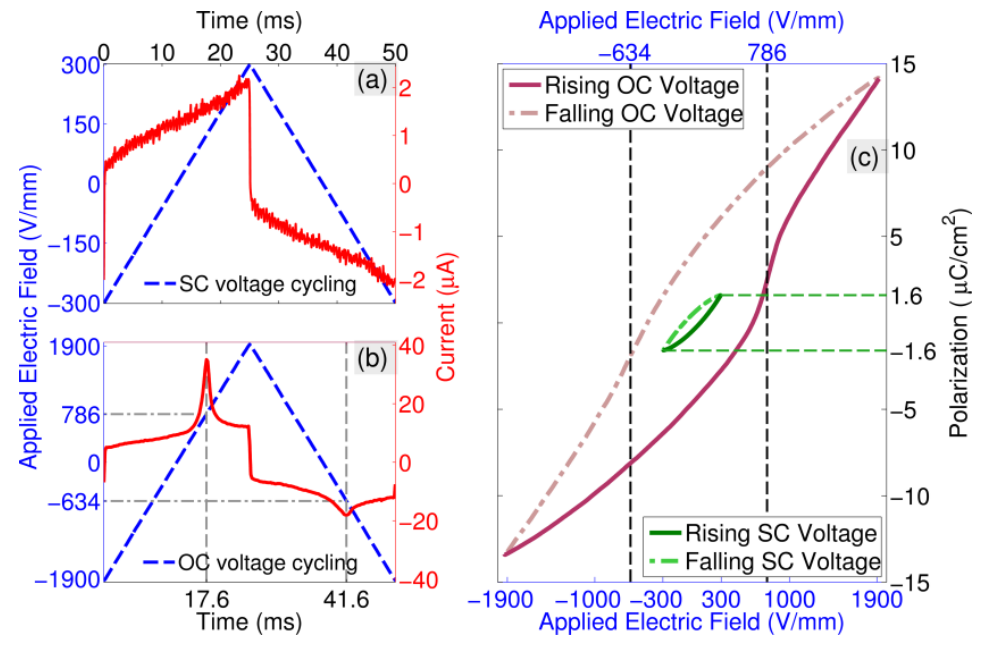

Figure 1. Dielectric responses of SBN50: (a,b): time-dependence of electric fields and currents for the case of sub-coercive and overcoercive fields (c): polarization-field loops. The magnitudes of coercive fields $(+786 /-634 \mathrm{~V} / \mathrm{mm})$ are marked on the axes.

The time-resolved X-ray diffraction experiment was performed using a custom-built stroboscopic data-acquisition system, which operates on the principle of a multi-channel analyser and qualifies for the investigation of repetitive processes down to the nanosecond time scale. The details of this technique are described elsewhere [4], [33-35] and briefly summarized in the supplemental materials. It has already been applied to the determination of small $\left(\sim 10^{-4} \AA\right)$ electric field induced bond distortions [36-42], the determination of piezoelectric coefficients [43], and the study of domain wall motion in ferroelectric ceramics [7], [30,44-47]. Our measurements were conducted at the P09 beamline at the 
PETRA III storage ring (Hamburg, Germany) using a six-circle diffractometer and a large dynamic range APD detector [48]. We tuned the X-ray wavelength to $\lambda=0.86 \AA$ to maximize the penetration depth to $10-20 \mu \mathrm{m}$ (depending on the reflection geometry). Then we analysed the dynamics of several rocking curves (RC) remembering that their angular positions depend on lattice parameters and a crystal orientation, while the shapes account for their distribution throughout the sample. As shown elsewhere [43,49] a homogeneous linear strain shifts a mass centre (MC) of RC by the following amount:

$$
\Delta \omega=-\tan \theta \cdot x_{i j} q_{i} q_{j}-x_{i j} y_{i} q_{j}+\Delta \omega_{r o t}
$$

Here, $\boldsymbol{x}$ is the strain tensor [50], $\theta$ is the Bragg angle, $\boldsymbol{q}$ and $\boldsymbol{y}=[\boldsymbol{w}, \boldsymbol{q}]$ are unit vectors: $\boldsymbol{q}$ and $\boldsymbol{w}$ point towards the corresponding reciprocal lattice node and diffractometer rotation axis correspondingly. All the vector and tensor components refer to the axes of crystal physical Cartesian system, aligned with the tetragonal crystallographic axes. $\Delta \omega_{\text {rot }}$ describes the field induced rotation of a sample. When a series of harmonic reflections, $n h n k n l$, is measured, Eq. 1 simplifies as [43]:

$$
\Delta \omega=A \cdot \tan \theta+B
$$

for which $A=-x_{i j} q_{i} q_{j}$ and $B$ are constant. We measured the shifts of the $00 l$ RCs, evaluated their $\tan \theta$ dependence, and calculated the $x_{33}=-A$ strain component (elongation of the lattice along the polar axis).

Figure 2 summarizes the dynamics of the $007 \mathrm{RC}$ during $20 \mathrm{~Hz}$ weak field cycling: figure 2(a) maps the time-dependence of the RC, Figures 2(b,c) shows the RCs collected within four different time channels, Figure 2(d) compares the positions of the MC with the RC maximum position (MP). Here, the MPs were located by a parabolic fit to the seven top points of the RCs. The systematic sliding of the RCs indicates the development of a time and field dependent deformation field. The observed RCs are asymmetric: their MCs are shifted to the left / right of the MPs at positive / negative voltages correspondingly. This asymmetry changes systematically with the electric field (Figure 2d), suggesting that each RC may sum up from two components moving in the opposite directions. Such movement is consistent with the electric field induced strain in ferroelectric domains with antiparallel polarization whose piezoelectric coefficients have opposite signs. However, we could not separate the RC profiles into two components because of their extreme proximity. 



Figure 2. Time-resolved X-ray diffraction on SBN50 under SC electric field cycling. (a) False-colour map of the $007 \mathrm{RC}$ as a function of time and relative rocking angle. (b, c) $007 \mathrm{RCs}$ corresponding to four selected time channels. The dashed lines mark their MC to highlight the RCs asymmetry changes. (d) Time dependence of the 007 RCs position: MC and MP. e) The amplitude of MC displacement of $00 /(I=3 \ldots 7)$ RCs as a function of $\tan \theta$.

The RCs of other $00 l(l=3 \ldots 7)$ Bragg reflections showed the same dynamics (see the supplemental materials). Therefore we applied equation (2) to calculate $x_{33}$ strain component averaged over the domains in the X-ray beam. The linear regression of the amplitude of MC displacement as a function of $\tan \theta$ in Figure 2(e) proves the validity of the approach. The arrows in Figure 2(d) mark the evaluated piezoelectric coefficients $d_{33}=\frac{\partial x_{33}}{\partial E_{3}}$ (in units of $\mathrm{pC} / \mathrm{N}$ ) during two selected time intervals. They clearly feature the non-linearity of the strainfield dependence: the $\left|d_{33}\right|=65 \mathrm{pC} / \mathrm{N}$ at $150 \mathrm{~V}$ exceeds the $41 \mathrm{pC} / \mathrm{N}$ at $0 \mathrm{~V}$ by $\sim 60 \%$. This situation differs from the strictly linear strain-field dependence in ordinary piezoelectrics, such as $\alpha$-quartz.

Figure 3 summarizes the dynamics of $007 \mathrm{RC}$ under $20 \mathrm{~Hz}$ triangular OC voltage cycling; it is organized similarly to Figure 2a-c. As in the weak field case, the RCs are asymmetric. The most interesting feature here is the clear separation of some RCs into one sharp and one broad component. The sharp component is confined within the angular interval $\sim[-10,10]$ mdeg and persists in the entire time range. The broad component spreads over $~[-40,-10]$ mdeg; it appears for the negative voltages only (e.g. RC1 / RC6 in Figure 3b /3c); its full width at half maxima (FWHM) depends on the electric field. In general, FWHM may vary due to changes of domain size or mosaicity of a crystal [51], [52]. However, as there is no physical reason 
for periodic mosaicity changes under electric field, we assume that the narrower / broader peaks originate from the volumes with larger ferroelectric domains (Volumes 1) / smaller ferroelectric domains (Volumes 2). From the angular positions of these peaks we concluded that the $c$-lattice parameter of the Volumes 2 is elongated relatively to that of the Volumes 1 .
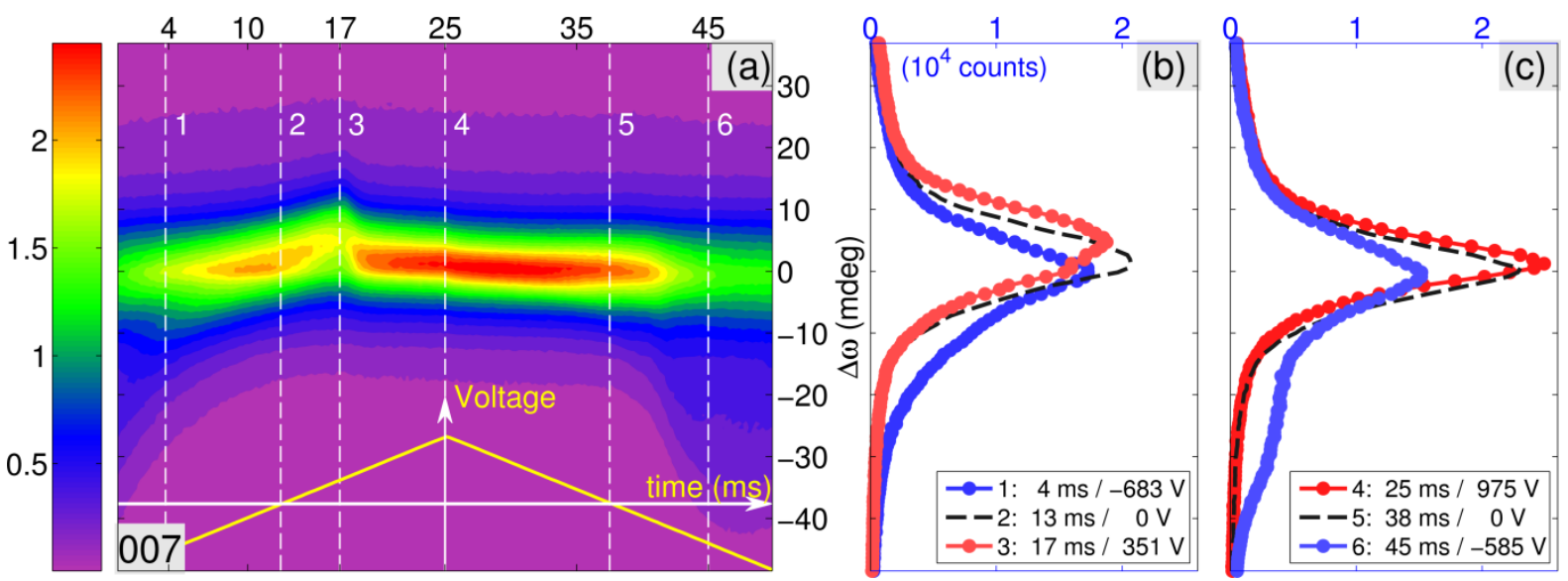

Figure 3. Summary of time-resolved X-ray diffraction of the 007 Bragg RC under OC electric field cycling. The figure is organized identically to Figure 2a-c.

The other important features are the rapid accelerations of the peak positions (MC and MP) at $\sim 15$ and $42 \mathrm{~ms}$ : their timing correlates with the maxima of the switching current (Figure 1). The other $00 l$ Bragg peaks exhibit qualitatively similar dynamics, but (as in the SC case), the amplitude of the MC motion increases with $l$ (suppl. material).

Figure 4 demonstrates the result of fitting the RCs with the sum of two symmetrical pseudoVoigt components [53] wherever such univocal separation was possible. The representative curves, demonstrating the quality of fitting, are available in the supplemental material. Figure 4a follows the time dependences of FWHM, the ratio of integrated intensities of broad and sharp peak, while Figure $4 \mathrm{~b}$ shows positions of their MCs. The gaps (where the univocal separation is impossible) are filled with the MC, MP, and FWHM of the RCs. The current is replicated for the identification of the switching intervals, marked as $\mathbf{S}+$ and $\mathbf{S}-$ - We will discuss the peak dynamics during these intervals.

a) $\mathbf{S}+$ features the polarization reversal induced by the positive voltage, i.e. from $\mathbf{P} \uparrow \downarrow \mathbf{c}$ to $\mathbf{P} \uparrow \uparrow \mathbf{c}$. The RCs profiles are asymmetric; their separation into two components is possible near the switching event only. The switching dynamics appears as a kink of the peak 2 position (Fig 3b) - this peak must represent those volumes which undergo switching, while the rest are frozen. Passing of the MC over a local maximum corresponds to the passing of the average $c$ lattice parameter over a local minimum. We also note that the FWHMs of both peaks remain constant upon this switching: this indicates that the polarization reverts without nucleation of 
small domains. Moreover, we assume that nearly a single domain state is generated at the highest positive voltage because it corresponds to the sharpest RC. This scenario is roughly consistent with the electrostriction law: $x_{33}(E)=Q_{d} \cdot P_{d}^{2}(E)$ where $P_{d}$ and $Q_{d}$ is the single domain polarization and electrostriction coefficient, respectively.
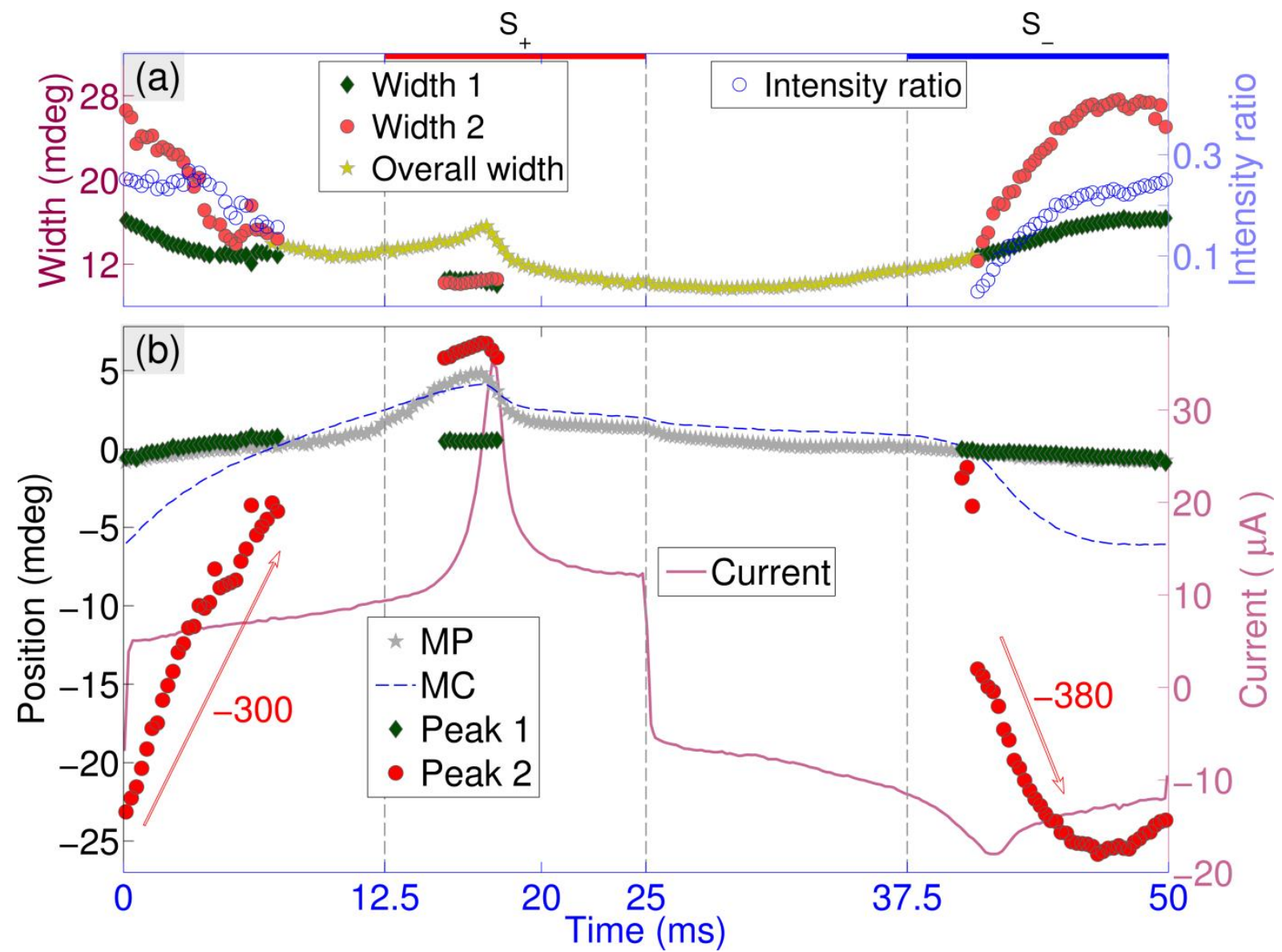

Figure 4. The dynamics of width, position and intensity ratios of peaks adding up to the $007 \mathrm{RC}$. The data are given wherever the univocal separation is feasible. Diamonds / circles correspond the dominating / weaker components. We also show the MP and MC positions and electrical current for the reference. The lines on the top highlight two different switching intervals ( $\mathbf{S}+$ and $\mathbf{S}-)$ discussed in the text.

b) $\mathbf{S}$ - represents the polarization reversal induced by the negative voltage, i.e. from $\mathbf{P} \uparrow \uparrow \mathbf{c}$ to $\mathbf{P} \uparrow \downarrow \mathbf{c}$. The dynamics of this reversal appears as a rapid detachment of the broad peak from the sharp peak and increasing of the FWHMs of both peaks, indicating the nucleation of small domains. Simultaneously, the broad peak rapidly moves towards lower Bragg angles and passes over their local minimum. This means that the average $c$-lattice parameter of the Volumes 2 must pass over a local maximum. The switching must be incomplete because the single domain state (as for $\mathbf{S}+$ ) with negative polarization is not reached. 


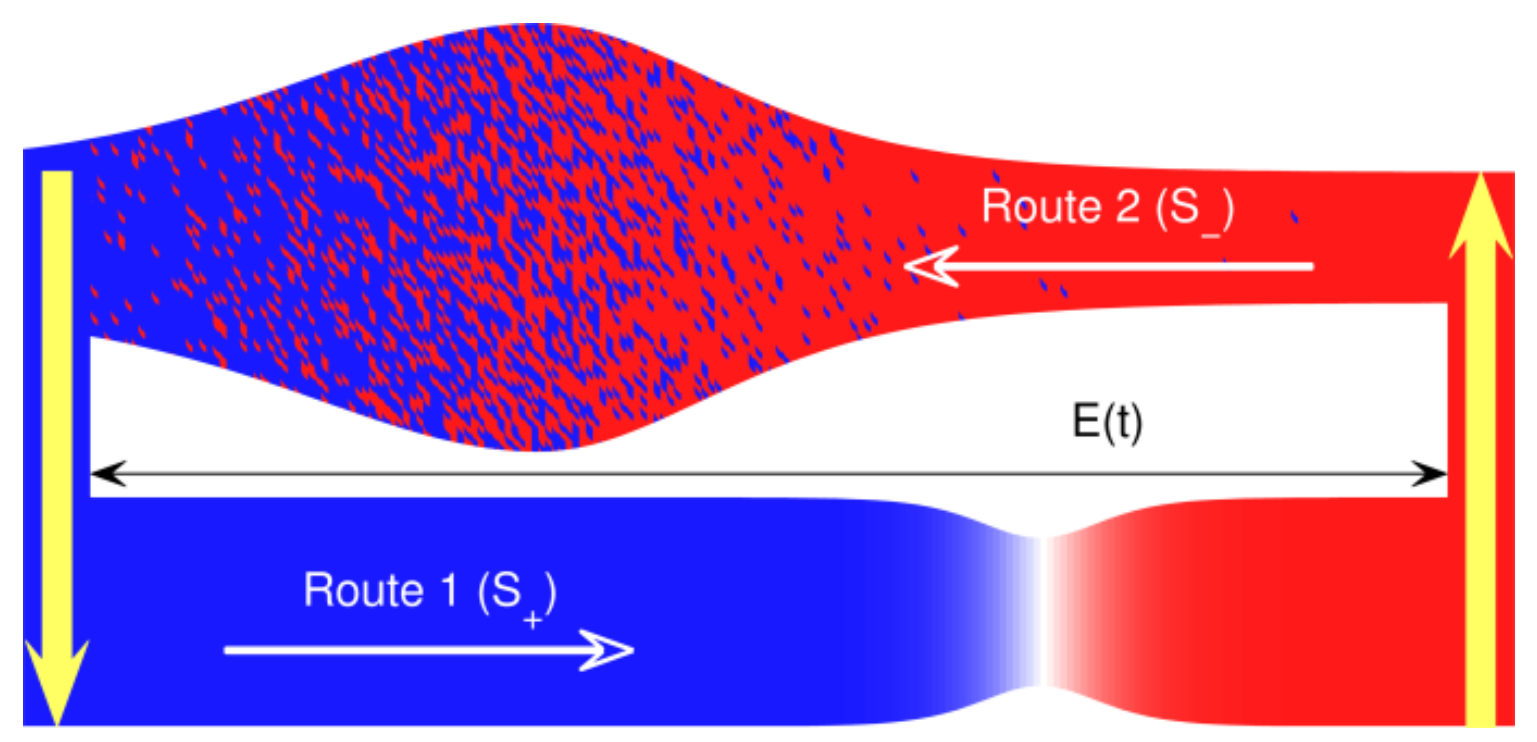

Figure 5. Schematics of the $\mathbf{S}+$ and $\mathbf{S}$ - polarization reversal routes. The colours map the regions of different polarizations: $\mathbf{P} \uparrow \uparrow \mathbf{c}$ (red), $\mathbf{P} \uparrow \downarrow \mathbf{c}$ (blue) or $\mathrm{P}=0$ (white). Single domain states are represented by the vertical arrows. S+ (bottom) illustrates the bulk-type switching, passing over the 'neck' (minimum of strain). S- (top) illustrates the switching via formation of small domains, passing over the 'waist' (maxima of strain).

These two switching routes follow completely different strain-field dependence, schematically illustrated in Figure 5. Two time intervals (marked by the arrows) in Figure 4 exhibit the fastest shift of the broad peak, witnessing enormously strong piezoelectricity in Volumes 2. The corresponding $\left|d_{33}\right|$ piezoelectric coefficients, estimated from the dynamics of 007, 006 and $005 \mathrm{RCs}$ are equal to $(300 \pm 100) \mathrm{pC} / \mathrm{N}$ (left arrow) and $(380 \pm 20) \mathrm{pC} / \mathrm{N}$ (right arrow), i.e. $\sim 6$ times higher than for the case of SC field. This piezoelectric activity is comparable with the one in PZT.

It is interesting that, like in the case of PZT [54], enhanced piezoelectricity is connected to the high density of small domains. We stress, however, that piezoelectricity in PZT and other perovskite-based materials is usually related to principally different mechanisms. They exploit the pseudocubic character of perovskites, in which the paraelectric-to-ferroelectric phase transition may generate strain and polarization domains, create competing phases of different symmetry [12], or even activate polarization rotation routes [55,56]. None of these opportunities exist in SBN50, where both phases are strictly tetragonal (c/a 0.31).

In summary, this Letter offers a new look on piezoelectricity and polarization reversal in ferroelectrics. Benefiting from the ability to follow lattice parameter(s) under an alternating electric field, we established that spontaneous polarization may be reverted via two substantially different routes passing over the minimum or over the maximum of strain. The 
first route is dominated by the bulk polarization reversal. The second route involves the nucleation of small inversion domains and exhibits a dramatic enhancement of the piezoelectric activity stemming from the correlation between the domain sizes and their lattice parameter. Thus, the important message of this Letter is that a colossal piezoelectric activity may arise from a mechanism which does not require any other ferroic ordering than the ferroelectric one. The possibility to create conditions, activating this mechanism in the entire material and at weak electric field, may open a new way of engineering high performance piezoelectrics. These conditions must be further investigated by e.g. in-situ real space domain mapping [9], [23], [57]. Thus, our results extend the list of possible connections between ferroic order and functional properties of materials.

\section{Acknowledgements}

This work is supported by German Federal Ministry of Education and Research (BMBF Bundesministerium für Bildung und Forschung - Grant Number 05K13PSA). We acknowledge Stefan Heidbrink for his help with the data acquisition system, the staff of P09 beamline at PETRA III for their support during the experiment, and Boriana Mihailova for stimulating discussions.

\section{References}

[1] W. Wersing, W. Heywang, H. Beige, and H. Thomann, in Piezoelectricity (SpringerVerlag, Berlin, 2008), pp. $37-87$.

[2] L. Bellaiche and D. Vanderbilt, Phys. Rev. Lett. 83, 1347 (1999).

[3] L. Bellaiche, A. Garcia, and D. Vanderbilt, Phys. Rev. Lett. 84, 5427 (2000).

[4] S. Gorfman, O. Schmidt, V. G. Tsirelson, M. Ziolkowski, and U. Pietsch, Zeitschrift Für Anorg. Und Allg. Chemie 639, 1953 (2013).

[5] X. Ren, Nat. Mater. 3, 91 (2004).

[6] S. Li, W. Cao, and L. E. Cross, J. Appl. Phys. 69, 7219 (1991).

[7] A. Pramanick, D. Damjanovic, J. E. Daniels, J. C. Nino, and J. L. Jones, J. Am. Ceram. Soc. 94, 293 (2011).

[8] E. Soergel, Appl. Phys. B 81, 729 (2005). 
[9] V. Y. Shur, V. A. Shikhova, A. V. Ievlev, P. S. Zelenovskiy, M. M. Neradovskiy, D. V. Pelegov, and L. I. Ivleva, J. Appl. Phys. 112, 064117 (2012).

[10] B. Noheda, D. E. Cox, G. Shirane, J. A. Gonzalo, L. E. Cross, and S.-E. Park, Appl. Phys. Lett. 74, 2059 (1999).

[11] B. Noheda, D. E. Cox, G. Shirane, R. Guo, B. Jones, and L. E. Cross, Phys. Rev. B 63, 014103 (2000).

[12] D. Phelan, X. Long, Y. Xie, Z.-G. Ye, A. M. Glazer, H. Yokota, P. A. Thomas, and P. M. Gehring, Phys. Rev. Lett. 105, 207601 (2010).

[13] S. Gorfman, D. S. Keeble, A. M. Glazer, X. Long, Y. Xie, Z.-G. Ye, S. Collins, and P. A. Thomas, Phys. Rev. B 84, 020102(R) (2011).

[14] G. Xu, Z. Zhong, Y. Bing, Z.-G. Ye, C. Stock, and G. Shirane, Phys. Rev. B 67, $104102(2003)$.

[15] G. Xu, Z. Zhong, Y. Bing, Z.-G. Ye, C. Stock, and G. Shirane, Phys. Rev. B 70, 064107 (2004).

[16] K. H. Conlon, H. Luo, D. Viehland, J. F. Li, T. Whan, J. H. Fox, C. Stock, and G. Shirane, Phys. Rev. B 70, 172204 (2004).

[17] C. Stock, G. Xu, P. M. Gehring, H. Luo, X. Zhao, H. Cao, J. F. Li, D. Viehland, and G. Shirane, Phys. Rev. B 76, 064122 (2007).

[18] G. Xu, Z. Zhong, Y. Bing, Z.-G. Ye, and G. Shirane, Nat. Mater. 5, 134 (2006).

[19] A. Bosak, D. Chernyshov, S. Vakhrushev, and M. Krisch, Acta Crystallogr. A. 68, 117 (2012).

[20] G. Esteves, C. M. Fancher, and J. L. Jones, J. Mater. Res. 1 (2014).

[21] S. Podlozhenov, H. A. Graetsch, J. Schneider, M. Ulex, M. Wöhlecke, and K. Betzler, Acta Crystallogr. B. 62, 960 (2006).

[22] V. V. Shvartsman, W. Kleemann, T. Łukasiewicz, and J. Dec, Phys. Rev. B 77, 054105 (2008).

[23] K. Matyjasek, J. Dec, S. Miga, and T. Łukasiewicz, Condens. Matter Phys. 16, 31701 (2013).

[24] V. V. Shvartsman, J. Dec, S. Miga, T. Łukasiewicz, and W. Kleemann, Ferroelectrics 376, 1 (2008).

[25] B. Jaffe, W. J. R. Cook, and H. Jaffe, Piezoelectric Ceramics (Academic Press, 1971).

[26] B. Jaffe, J. Appl. Phys. 25, 809 (1954). 
[27] T. Lukasiewicz, M. A. Swirkowicz, J. Dec, W. Hofman, and W. Szyrski, J. Cryst. Growth 310, 1464 (2008).

[28] D. V. Taylor and D. Damjanovic, J. Appl. Phys. 82, 1973 (1997).

[29] D. Damjanovic, J. Appl. Phys. 82, 1788 (1997).

[30] A. Pramanick, D. Damjanovic, J. C. Nino, and J. L. Jones, J. Am. Ceram. Soc. 92, 2291 (2009).

[31] T. Woike, T. Volk, U. Dörfler, R. Pankrath, and L. I. Ivleva, Ferroelectr. Lett. 23, 127 (1998).

[32] V. V Gladki, V. A. Kirikov, and T. Rt. Volk, Magn. Ferroelectr. 44, 351 (2002).

[33] S. Gorfman, O. Schmidt, M. Ziolkowski, M. Kozierowski, and U. Pietsch, J. Appl. Phys. 108, (2010).

[34] S. Gorfman, Crystallogr. Rev. 20, 210 (2014).

[35] G. Eckold, H. Gibhardt, D. Caspary, P. Elter, and K. Elisbihani, Zeitschrift Für Krist. 218, 144 (2003).

[36] V. G. Tsirelson, S. Gorfman, and U. Pietsch, Acta Crystallogr. A. 59, 221 (2003).

[37] R. Guillot, P. Fertey, N. K. Hansen, P. Allé, E. Elkaïm, and C. Lecomte, Eur. Phys. J. B 42, 373 (2004).

[38] S. Gorfman, V. G. Tsirelson, and U. Pietsch, Acta Crystallogr. A. 61, 387 (2005).

[39] R. Guillot, P. Allé, P. Fertey, N. K. Hansen, and E. Elkaïm, J. Appl. Crystallogr. 35, 360 (2002).

[40] O. Schmidt, S. Gorfman, and U. Pietsch, Cryst. Res. Technol. 43, 1126 (2008).

[41] O. Schmidt, S. Gorfman, L. Bohatý, E. Neumann, B. Engelen, and U. Pietsch, Acta Crystallogr. A. 65, 267 (2009).

[42] P. Fertey, P. Allé, E. Wenger, B. Dinkespiler, O. Cambon, J. Haines, S. Hustache, K. Medjoubi, F. Picca, A. Dawiec, P. Breugnon, P. Delpierre, C. Mazzoli, and C. Lecomte, J. Appl. Crystallogr. 46, 1151 (2013).

[43] S. Gorfman, O. Schmidt, U. Pietsch, P. Becker, and L. Bohatý, Zeitschrift Für Krist. 222, 396 (2007).

[44] J. L. Jones, M. Hoffman, J. E. Daniels, and A. J. Studer, Appl. Phys. Lett. 89, 092901 (2006).

[45] J. L. Jones, A. Pramanick, J. C. Nino, S. Maziar Motahari, E. Üstündag, M. R. Daymond, and E. C. Oliver, Appl. Phys. Lett. 90, 172909 (2007). 
[46] A. Pramanick, J. E. Daniels, and J. L. Jones, J. Am. Ceram. Soc. 92, 2300 (2009).

[47] A. Pramanick, A. D. Prewitt, M. a. Cottrell, W. Lee, A. J. Studer, K. An, C. R. Hubbard, and J. L. Jones, Appl. Phys. A 99, 557 (2010).

[48] J. Strempfer, S. Francoual, D. Reuther, D. K. Shukla, A. Skaugen, H. SchulteSchrepping, T. Kracht, and H. Franz, J. Synchrotron Radiat. 20, 541 (2013).

[49] H. Graafsma, J. Appl. Crystallogr. 25, 372 (1992).

[50] F. Nye, Physical Properties of Crystals and Their Representation by Tensors and Matrices (Clarendon Press, Oxford, 1985).

[51] B. E. Warren, X-Ray Diffraction (Dover publications, New York, 1990).

[52] P. J. Becker and P. Coppens, Acta Crystallogr. Sect. A 30, 129 (1974).

[53] R. J. Angel, J. Appl. Crystallogr. 36, 295 (2003).

[54] K. A. Schönau, M. Knapp, H. Kungl, M. J. Hoffmann, and H. Fuess, Phys. Rev. B 76, 144112 (2007).

[55] D. S. Keeble, E. R. Barney, D. A. Keen, M. G. Tucker, J. Kreisel, and P. A. Thomas, Adv. Funct. Mater. 23, 185 (2013).

[56] N. Zhang, H. Yokota, A. M. Glazer, Z. Ren, D. A. Keen, D. S. Keeble, P. A. Thomas, and Z.-G. Ye, Nat. Commun. 5, 5231 (2014).

[57] V. Y. Shur, V. A. Shikhova, P. S. Zelenovskiy, D. V. Pelegov, L. I. Ivleva, and J. Dec, J. Adv. Dielectr. 04, 1450006 (2014). 